\title{
Stuck in a lockdown: dreams, bad dreams, nightmares, and their relationship to stress, depression and anxiety during the COVID-19 pandemic.
}

Elizaveta Solomonova*1,2, Claudia Picard-Deland ${ }^{3,4}$, Iris Rapoport ${ }^{5}$, Marie-Hélène Pennestri ${ }^{6,7}$, Mysa Saad ${ }^{8}$, Tetyana Kendzerska9 ${ }^{9}$, Samuel Paul Louis Veissiere ${ }^{2}$, Roger Godbout ${ }^{7,11}$, Jodi D. Edwards $^{10,12}$, Lena Quilty ${ }^{13}$, Rebecca Robillard ${ }^{8,14}$

${ }^{1}$ Neurophilosophy Lab, McGill University

${ }^{2}$ Culture, Mind and Brain research group; Division of Social and Transcultural Psychiatry, Department of Psychiatry, McGill University

${ }^{3}$ Dream and Nightmare Laboratory, Center for Advanced Research in Sleep Medicine

${ }^{4}$ Department of Neuroscience, University of Montreal

${ }^{5}$ Department of Philosophy, McGill University

${ }^{6}$ Department of Educational and Counselling Psychology, McGill University

${ }^{7}$ Sleep Laboratory \& Clinic, Hôpital en santé mentale Rivière-des-Prairies, CIUSSS du Nord-del'Île-de-Montréal

${ }^{8}$ The Royal's Institute of Mental Health Research

${ }^{9}$ Department of Medicine, The Ottawa Hospital Research Institute, University of Ottawa

${ }^{10}$ University of Ottawa Heart Institute

${ }^{11}$ Department of Psychiatry, University of Montreal

${ }^{12}$ Centre for Addiction and Mental Health, Department of Psychiatry, University of Toronto

${ }^{13}$ School of Epidemiology and Public Health, University of Ottawa

${ }^{14}$ School of Psychology, University of Ottawa

\section{Target journal: PLOS One}

\author{
*Corresponding author \\ Elizaveta Solomonova, $\mathrm{PhD}$ \\ Division of Social and Transcultural Psychiatry \\ McGill University \\ Montreal, Quebec, Canada \\ elizaveta.solomonova@mail.mcgill.ca
}




\section{Abstract}

Background: An upsurge in dream and nightmare frequency has been noted since the beginning of the COVID-19 pandemic and research shows increases in levels of stress, depression and anxiety during this time. Growing evidence suggests that dream content has a bidirectional relationship with psychopathology, and that dreams react to new, personally significant and emotional experiences. The first lockdown experience was an acute event, characterized by a combination of several unprecedent factors (new pandemic, threat of the disease, global uncertainty, , experience of social isolation, exposure to stressful information) that resulted in a large-scale disruption of life routines.

This study aimed at investigating changes in dream, bad dream and nightmare recall; most prevalent dream themes, and the relationship between dreams, bad dreams, nightmares and symptoms of stress, depression and anxiety during the first COVID-19 lockdown (April-May 2020) through a national online survey.

Methods: 968 participants completed an online survey. Dream themes were measured using the Typical Dreams Questionnaire; stress levels were measured by the Cohen's Perceived Stress Scale; symptoms of anxiety were assessed by Generalized Anxiety Disorder (GAD-7) scale; and symptoms of depression were assessed using the Quick Inventory of Depressive Symptomatology.

Results: 34\% (328) of participants reported increased dream recall during the lockdown. The most common dream themes were centered around the topics of 1) inefficacy (e.g., trying again and again, arriving late), 2) human threat (e.g., being chased, attacked); 3) death; and 4) pandemic imagery (e.g., being separated from loved ones, being sick). Dream, bad dream and nightmare frequency was highest in individuals with moderate to severe stress levels. Frequency of bad dreams nightmares and dreams about the pandemic, inefficacy and death were associated with higher levels of stress, as well as with greater symptoms of depression and anxiety.

Conclusions: Results support theories of dream formation, environmental susceptibility and stress reactivity. Dream content during the lockdown broadly reflected existential concerns and was associated with increased symptoms of mental health indices.

Keywords: dreams, nightmares, covid-19 pandemic, stress, anxiety, depression, inefficacy 


\section{Introduction}

Since the declaration of the global COVID-19 pandemic in March 2020 by the World Health Organization and since measures of social isolation have been in place in many countries, including Canada, dreams have been a hot topic of conversation. A number of popular outlets reported an increase in dream reports, an increase in bizarre, threatening dreams (Carey, 2020; Collie, 2020; Rani, 2020; Valenti, 2020; Weaver, 2020), and social media became saturated with reports of new dream experiences, all related to the elements of COVID-19 - a phenomenon that Nielsen called "a dream surge" (Nielsen, 2020). In line with renewed interest in dreams during the pandemic, and following early anecdotal evidence for intensified dreaming, growing research provides evidence for specific changes in dream content during the acute stage of the health crisis. Dreams, currently defined as any cognitive activity that happens during sleep which has a subjective component that is recalled in wake (Nielsen, 2000), are known to react to most pressing concerns, both of personal and collective nature, and are thought to play a role in the integration of lived experiences, in extracting the gist of new information and in emotion regulation (Walker \& van der Helm, 2009). The current pandemic offers a unique opportunity to investigate changes to our oneiric lives brought about by shared concerns during the times of dealing with illness/threat of illness and in context of unprecedented degree of change in socioeconomic aspects of life. In this paper, we present results of an ongoing national online survey focused on multiple facets of mental health, social and economic impacts of COVID-19 and including validated sleep and dream questionnaires. Our project had three goals: 1) to qualify changes in dream, bad dream and nightmare recall; 2) to investigate most prevalent dream themes; and 3) to assess the relationship between dreams, stress, and symptoms of anxiety and depression during the first lockdown phase of COVID-19.

\subsection{COVID-19, sleep and mental health}

An emerging body of research shows the many ways in which the experience of the pandemic, and in particular of social isolation, is affecting sleep specifically and mental health more broadly, notably with documented increases and exacerbations in symptoms of depression and anxiety (Gasteiger et al., 2021; Kornilaki, 2021; Mautong et al., 2021; Robillard, Daros, et al., 2021; Robillard, Saad, et al., 2020; Salari et al., 2020). Mounting evidence shows widespread 
changes in sleep patterns, including increased sleep duration, later bedtime and later awakenings, as well as a reduction in social jet lag (a form of circadian misalignment between one's endogenous best rhythm and demands of the social world, such as early work or school start)

(Blume, Schmidt, \& Cajochen, 2020; Cellini, Canale, Mioni, \& Costa, 2020; Kantermann, 2020; Leone, Sigman, \& Golombek, 2020; Robillard, Dion, et al., 2021; Wright et al., 2020). In a number of studies, increased sleep time and opportunities for sleep schedules more aligned with one's chronotype, nevertheless, did not preclude reports of poor sleep quality, likely due to increased levels of stress associated with pandemic, lockdown, and personal challenges (Casagrande, Favieri, Tambelli, \& Forte, 2020; Cellini et al., 2020; Huang \& Zhao, 2020; Leone et al., 2020). Unsurprisingly, then, increased rates of insomnia were also reported by multiple teams (Gualano, Lo Moro, Voglino, Bert, \& Siliquini, 2020; Marelli et al., 2020; Voitsidis et al., 2020). Using data from the larger cohort of the current study, our group has previously reported an important increase in stress levels during the pandemic, a relationship between increased stress and pre-existing mental illness (Robillard, Daros, et al., 2021; Robillard, Saad, et al., 2020), as well as changes in patterns of sleep duration (increase vs. decrease) and timing (delay) during the pandemic (Robillard, Dion, et al., 2020). The role of disordered sleep in exacerbating mental disorders, in particular, depression and anxiety, is well documented (Alvaro, Roberts, \& Harris, 2013). During confinement, poor sleep was similarly associated with higher levels of psychotic-like experiences, rumination and somatic symptoms (Simor et al., 2020). This bidirectional relationship between sleep and mental health may also be evidenced by dysphoric or intensified dreaming.

\subsection{Dream content: mental health and relationship to lived experiences}

Dream content is often seen as a proxy for understanding individual experience of health and distress: dreams are known to react to new experiences and life changes. Bad dreams and nightmares tend to increase during periods of high stress (Carr \& Nielsen, 2017; Nielsen, 2017; Schredl et al., 2019), and are often exacerbated by, and potentially play a role in, flare-ups of mental health issues, including depression (Marinova et al., 2014) and anxiety (Spoormaker, Schredl, \& van den Bout, 2006). In general, many current theories of dream formation and of dream function converge on the following main ideas. It is suggested that dream content: 1) reflects elements of daily lived experience (Eichenlaub, Cash, \& Blagrove, 2017; Malinowski \& 
Horton, 2014; Nielsen \& Stenstrom, 2005); 2) extracts its meaning by creatively integrating new information into existing autobiographical memory networks (Hartmann, 1996, 2010; Horton \& Malinowski, 2015; Malinowski \& Horton, 2015; Wamsley \& Stickgold, 2010); and 3) preferentially responds to current concerns and to emotionally charged events with high personal significance (Malinowski \& Horton, 2014; van Rijn et al., 2015).

In addition to individual experiences, collective trauma can affect the content and intensity of dreams in the general population. For example, studies tracking dreams before and after the 9/11 attacks observed an increase in nightmare frequency in male respondents (Nielsen, Stenstrom, \& Levin, 2006), an increase in the intensity of the dream imagery (Hartmann \& Brezler, 2008) and clear incorporations of a variety of 9/11-related topics, such as planes crashing into buildings, being hijacked and bombs (Bulkeley \& Kahan, 2008). Similarly, studies tackling dream content in prisoners of wars and Holocaust survivors (Barrett et al., 2013;

Bergman et al., 2020) revealed a profound intrusion of war-related themes in dreams, as well as a long-lasting increase in dream negative emotions and threatening events. Lastly, a study on WWII Veterans (Sandman et al., 2013) showed more frequent nightmares and symptoms of insomnia, depression and anxiety that persisted even decades after the war.

The current pandemic, and in particular, the experience of a lockdown, presents a set of new challenges to our psychological and social health. These include novel experiences which have a strong emotional component, and of which we are, individually and collectively, attempting to make sense. It is therefore unsurprising that dreams should change during these times, as a process of making sense of and of adapting to these novel aspects of life. Thus, this historical period represents a unique and naturalistic opportunity to explore current theories of dream formation, including frameworks of environmental susceptibility and stress reactivity.

\subsection{Typical Dream Themes}

Previous work demonstrated a number of common themes in dream content. The Typical Dreams Questionnaire, first used in a sample of Canadian University students (Nielsen et al., 2003), investigates relative prevalence of different dream themes. This instrument has been validated in a German (Schredl, Ciric, Gotz, \& Wittmann, 2004) and a Chinese (Yu, 2008) samples. Results of the three studies converge on the possibility that dream themes are relatively stable across individual life span and are relatively similar across cultures, potentially 
representing shared concerns, fears and aspirations, common to members of the globalized world. The most common themes in dreams of the Canadian sample, specifically, were: being chased, sexual experiences, falling, school/studying and arriving too late (Nielsen et al, 2003). The current pandemic presents unique and new context for study of personal and collective experience. This novel context, characterized by such concerns and experiences as threat and experience of contagious disease, social isolation, personal and economic uncertainty, lockdowns, and others, quite unprecedentedly, is shared across most countries. We hypothesize that new dream themes will emerge as most prevalent during the lockdown in response to these pandemic-specific challenges.

\subsection{Dreams during COVID-19}

An increasing amount of studies report changes in dream content during the current pandemic. An upsurge of dream (Gorgoni et al., 2021; Schredl \& Bulkeley, 2020) and nightmare (Bruni et al., 2021; Giardino, Huck-Iriart, Riddick, \& Garay, 2020; Kilius, Abbas, McKinnon, \& Samson, 2021; Pesonen et al., 2020; Tu, He, \& Zhou, 2020) frequencies has been observed during lockdown, especially for the female sex (Kilius et al., 2021; Lehmann et al., 2021; Scarpelli et al., 2021), for individuals with sleep disturbances, anxiety or depressive symptoms (Gorgoni et al., 2021; Scarpelli et al., 2021) and for individuals with COVID-related increased in stress levels (Pesonen et al., 2020). An increase in nightmares, without COVID-19 imagery, was also observed in Canadian patients previously diagnosed with primary PTSD (Gupta, 2020). Further, the presence of COVID-19 related nightmares has been associated with symptoms of generalized anxiety disorder in healthcare providers in Colombia (Monterrosa-Castro, RedondoMendoza, \& Mercado-Lara, 2020). Similarly, the presence of COVID-19 related dreams in a large Spanish sample was associated with many factors, including having infected family or friends, reading news about coronavirus, and having higher depression, anxiety or stress scores (Dal Santo et al., 2021). With regards to specific features of dream content, Brazilian (Mota et al., 2020), U.S. (Barrett, 2020), Italian (Gorgoni et al., 2021; Iorio, Sommantico, \& Parrello, 2020; Scarpelli et al., 2021), Canadian (Kilius et al., 2021) and Chinese (Wang, Zemmelman, Hong, Feng, \& Shen, 2020) teams, all reported increases in negative dreams during the pandemic, including increase in themes of anxiety, sadness, aggressive interactions and preoccupations with health. Similarly, a U.S. survey revealed that $15 \%$ of the participants' 
dreams shifted towards more negative dream emotions (Schredl \& Bulkeley, 2020). Using automatic text algorithms on dream content during lockdown, Pesonen et al. (2020) found that a majority of distressing dreams were pandemic-specific, with themes such as failures in social distancing, coronavirus contagion, dystopia, and apocalypse. These dreams were more accentuated in individual with higher perceived stress and, while the imagery was pandemicspecific, they were nonetheless similar to typical idiopathic dream themes (e.g. failure, death). Lastly, in a sample of Canadian university students (MacKay \& DeCicco, 2020), dreams during the early weeks of the COVID-19 pandemic were characterized by increased virus imagery, as well as by animal imagery, and location changes.

\subsection{Study objectives and hypotheses}

The objectives of the current study were to characterize dream, bad dream and nightmare recall during the first lockdown phase of the COVID-19 pandemic; to identify most prevalent dream themes, and to investigate the relationship between dream and psychological variables, specifically: the degree of concern with relation to different facets of the pandemic, levels of stress, as well as symptoms of anxiety and depression. We hypothesized that: 1) dream, bad dream and nightmare recall would increase; 2) that pandemic- and threat-related imagery would be frequent and reflect ongoing concerns; 3) that higher dream, bad dream and nightmare recall, as well as the most prevalent dream themes, would be associated with higher levels of stress, with more concerns over COVID-19 and greater symptoms of depression and anxiety; and 4) that, keeping in mind the bi-directional nature of the relationship between dysphoric dreaming and psychopathology, bad dream and nightmare frequency as well as pandemic- and threatrelated imagery would in turn predict symptoms of stress, depression and anxiety.

\section{Materials and Methods}

\subsection{Survey}

We present here data from the ongoing longitudinal national "How are you coping" webbased survey. The survey aims at characterizing the many facets of impact of the covid-19 pandemic on different aspects of mental health. The full methodology of the survey can be found elsewhere (Robillard, Saad, et al., 2020). The study was approved by the Clinical Trials Ontario- 
Qualified Research Ethics Board (Protocol \#2131). Electronically informed consent was obtained from participants prior to starting the survey. The complete survey contained a main part, assessing psychiatric, social, economic and health impacts of the pandemic, and an optional component. Dream themes were included in the optional component.

\subsection{Participants}

A total of 968 out of XXX participants (12-92 years old, Mean age $=52.5 \pm 17.2$; Female $=710$, Male=258) completed the optional component of the survey between April 3 and May 15 2020. Most participants $(n=940)$ were located in Canada during the outbreak, 12 in the United States, 4 in France, 3 in the United Kingdom, 1 in Australia and 1 in New Zealand, and 7 declined to provide location. 135 (14\%) of the participants reported working in essential services (not closed during the lockdown), of which 13 worked in a hospital, 6 in a grocery store, 1 in public transportation, and 36 in other essential services.

\subsection{Measures}

Dream themes were assessed using a modified version of the Typical Dreams Questionnaire (TDQ) (Nielsen et al., 2003). In addition to the 56 themes from the original questionnaire, we added 4 items (germs or being contaminated, being in a hospital, being sick, and being separated from a loved one), representative of some of the major concerns during the pandemic. While the original TDQ focuses on lifetime prevalence of each theme, we asked participants to rate how often they have dreamt of each theme during the past week. Overall dream theme prevalence ( $\%$ of people endorsing each item at least once), and frequency of experience of each dream theme ( $0=$ "never", $1=$ "once", $2=$ "2-3 times", $3=$ " $4-10$ times", $4=$ "11+ times") were calculated. Individual dream themes were then combined into the following 7 larger topics: Pandemic, Natural Threat, Human Threat, Inefficacy, Paralysis, Death and Paranormal imagery. Prevalence for each topic was calculated as the percentage of people that dreamt at least once of an item included in that topic. The list of specific items that were used in each topics is presented in Table 1. 
Table 1. Dream topics and dream themes

\begin{tabular}{|c|c|}
\hline Dream topics & TDQ items \\
\hline Pandemic $^{\mathrm{a}}$ & $\begin{array}{l}\text { germs or being contaminated }(\# 57) \\
\text { being sick }(\# 58) \\
\text { being in hospital }(\# 59) \\
\text { being separated from a loved one }(\# 60)\end{array}$ \\
\hline Natural Threat & $\begin{array}{l}\text { snakes }(\# 9) \\
\text { flood or tidal waves }(\# 21) \\
\text { tornadoes or strong winds (\#22) } \\
\text { earthquakes }(\# 23) \\
\text { insects or spiders }(\# 24) \\
\text { fire }(\# 34) \\
\text { wild, violent beasts }(\# 40)\end{array}$ \\
\hline Human Threat & $\begin{array}{l}\text { being chased or pursued, but not physically injured (\#1) } \\
\text { being physically attacked (beaten, stabbed, raped, etc.) (\#2) } \\
\text { vividly sensing a presence in the room (\#29) }\end{array}$ \\
\hline Inefficacy & $\begin{array}{l}\text { trying again and again to do something (\#3) } \\
\text { arriving too late, e.g., missing a train (\#6) } \\
\text { being locked } u \mathrm{p}(\# 8) \\
\text { losing control of a vehicle (\#33) } \\
\text { failing an examination (\#38) }\end{array}$ \\
\hline Paralysis & $\begin{array}{l}\text { being frozen with fright }(\# 4) \\
\text { being tied, unable to move (\#15) } \\
\text { being smothered, unable to breathe (\#39) } \\
\text { being half awake and paralyzed in bed (\#44) }\end{array}$ \\
\hline Death & $\begin{array}{l}\text { being killed }(\# 27) \\
\text { seeing yourself as dead }(\# 28) \\
\text { a person now dead as alive }(\# 35) \\
\text { a person now alive as dead }(\# 36) \\
\text { killing someone }(\# 42)\end{array}$ \\
\hline Paranormal & $\begin{array}{l}\text { having superior knowledge or mental ability (\#16) } \\
\text { having magical powers (\#20) } \\
\text { seeing a UFO (\#46) } \\
\text { seeing extra-terrestrials ( } \# 47) \\
\text { traveling to another planet or visiting a different part of the universe (\#48) } \\
\text { being an animal (\#49) } \\
\text { seeing an angel (\#51) } \\
\text { encountering God in some form (\#52) } \\
\text { encountering a kind of evil force or demon (\#56) }\end{array}$ \\
\hline
\end{tabular}

${ }^{\mathrm{a}}$ Items not included in the original version of TDQ-56

Dream, bad dream and nightmare recall were assessed by asking participants 1) the average weekly number of dreams, bad dreams or nightmares they recalled in the past year preceding the lockdown, and 2) in the past 7 days.

Pandemic-related concerns were assessed using the following questions: participants were asked to rate how concerned they currently were about: 1) lacking food, 2) public services shutting down; 3) schools shutting down or staying closed for an extended period; 4) their 
children or relatives not coping well with the situation; and 5) not being able to access medications or medical services. Each type of concern was rated on a sliding scale from 0 (not concerned at all) to 100 (very concerned).

Participants were asked to rate their stress, anxiety and depression levels for the last 7 days. Stress levels were assessed using Cohen's Perceived Stress Scale (PSS (Cohen, Kamarck, $\&$ Mermelstein, 1983). Participants were then separated into low $(0=13)$ moderate $(14-25)$ or severe ( $>27)$ stress groups based on the PSS scores. Anxiety symptoms were evaluated using the Generalized Anxiety Disorder (GAD-7) 7-item scale (Spitzer, Kroenke, Williams, \& Lowe, 2006), and depressive symptoms were investigated using the Quick Inventory of Depressing Symptomatology (QIDS) scale (Rush et al., 2003). Sleep duration (in hours per day,) was assessed as part of the Pittsburgh Sleep Quality Index (PSQI) questionnaire (Buysse, Reynolds, Monk, Berman, \& Kupfer, 1989) adjusted to reflect two periods: 1) the last month before the outbreak, and 2) for the last 7 days.

\subsection{Statistical analyses}

Wicoxcon signed-rank related samples tests were used to assess change in dream recall, bad dream and nightmare frequency from pre-COVID-19 outbreak to the last 7 days. Descriptive statistics were used to assess prevalence of each individual dream theme and of broader dream topics. Spearman correlations were performed to investigate the relationship between the frequency of the most prevalent dream themes, level of concern for specific aspects of the pandemic, levels of stress, symptoms of anxiety and depression.

A MANCOVA with dream, bad dream and nightmare recall as dependent measures and with stress levels (low, medium, high) as independent factors, and age and sex as covariates, was performed to test whether dream variables were associated with stress levels. Follow up separate univariate ANOVAs were used to investigate potential differences in dream, bad dream and nightmare recall across stress levels (low, moderate, severe). A MANCOVA with most frequent dream topics as dependent variables, stress levels (low, moderate, severe) as independent factors and dream recall, age and sex as covariates was performed to test the relationship between stress group and dream topics. Separate follow up univariate ANCOVAs with stress levels (low, moderate and severe) as independent factors, dream recall as a covariate and frequency of four 
most prevalent dream topics as dependent factors were performed to assess the relationship between stress and specific dream topics.

Lastly, to investigate the potential predictor role for dream variables on mental health outcomes, we performed separate linear regressions with dream recall, bad dream and nightmare frequency as predictors; age, sex, income (above/below CAD 40,000 per year), employment status (employed/unemployed/retired) and antidepressant use as covariates; and stress, depression and anxiety levels as dependent variables.

\section{Results}

\subsection{Dream recall and dream themes}

\subsubsection{Dream, bad dream and nightmare recall}

A Wilcoxon signed-rank test revealed a weak decrease in dream recall from the year before the pandemic to the last 7 days $(z=4.491, p<.001)$. Mean dream recall was $3.26 \pm 4.97$ dreams per week in the year pre-pandemic, and $2.86 \pm 2.02$ dreams per week in the last 7 days. (Median before pandemic and in the last 7 days $=2$ dreams per week) 443 participants $(46 \%)$ reported no change in their dream recall (mean dream recall in the last 7 days $=2.73 \pm 1.99)$ as compared to past year. However, 328 (34\%) reported an increase (mean dream recall in the last 7 days $=3.49 \pm 2.05 ; 28 \%$ higher than no change group) and $188(19 \%)$ reported a decrease (mean dream recall in the last 7 days $=2.14 \pm 2.17 ; 22 \%$ lower than the no change group) in dream recall.

The weekly frequency of bad dreams decreased from the year before the pandemic to the last 7 days $(z=-5.799, p<.001)$. Mean bad dream recall was $1.56 \pm 4.71$ bad dreams per week in the year pre-pandemic, and $1.42 \pm 1.86$ bad dreams per week in the last 7 days. (Median before pandemic $=.5$, median in the last 7 days $=1$ bad dream per week).

The weekly frequency of nightmares, however, did not change (from past year to past 7 days, $z=1.540 . p=.123)$. Mean nightmare recall was $.82 \pm 2.16$ nightmares per week during the year pre-pandemic, and $.76 \pm 1.52$ nightmares per week during the last 7 days. 
There was no significant correlation between sleep duration and dream recall. However, weak correlations were revealed between shorter sleep duration and greater bad dream recall $\left(r_{s}=-.116\right.$, $p<.001)$, and nightmare recall $\left(r_{s}=-.178, p<.001\right)$.

\subsubsection{Dream themes and topics during the pandemic}

The following dream themes (individually, from 60 items of the modified TDQ) were the five most prevalent: 1) Trying over and over to do something (51.55\%); 2) Sexual experiences (41.32\%); 3) Arriving too late (32.95\%); 4) Being separated from a loved one (29.65\%); 5) Being chased or pursued (29.24\%). The four TDQ items that comprised the topic of the pandemic imagery, individually, had the following frequencies: 1) Being separated from a loved one (29.65\%); 2) Being sick (14.77\%); 3) Being in a hospital (12.00\%); 4) Germs and contamination (7.64\%). The frequencies of 25 most common themes are plotted in Figure 1.

When grouped by larger topics, the prevalence was as follows (\% of participants reporting dreaming of a theme at least once in the past 7 days): 1) Inefficacy $(62.40 \%)$; 2) Human threat (42.46\%); 3) Death (38.95\%); 4) Pandemic (37.19\%); 5) Paralysis (27.79\%); 6) Paranormal $(26.24 \%) ; 7)$ Natural threat (18.60\%). These results are presented in Figure 2. 


\section{TDQ individual items}

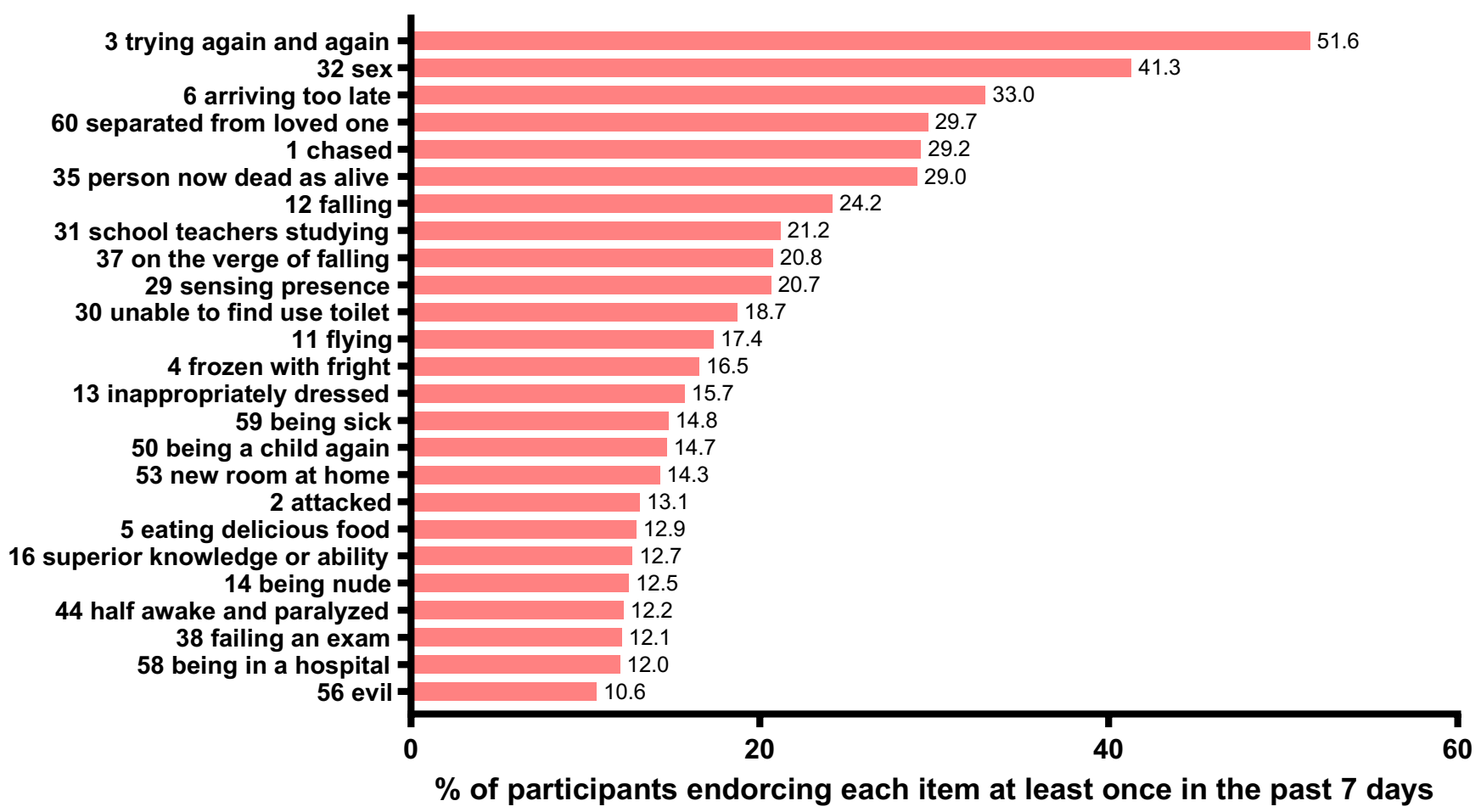

Figure 1. Frequency of 25 most prevalent individual TDQ dream themes

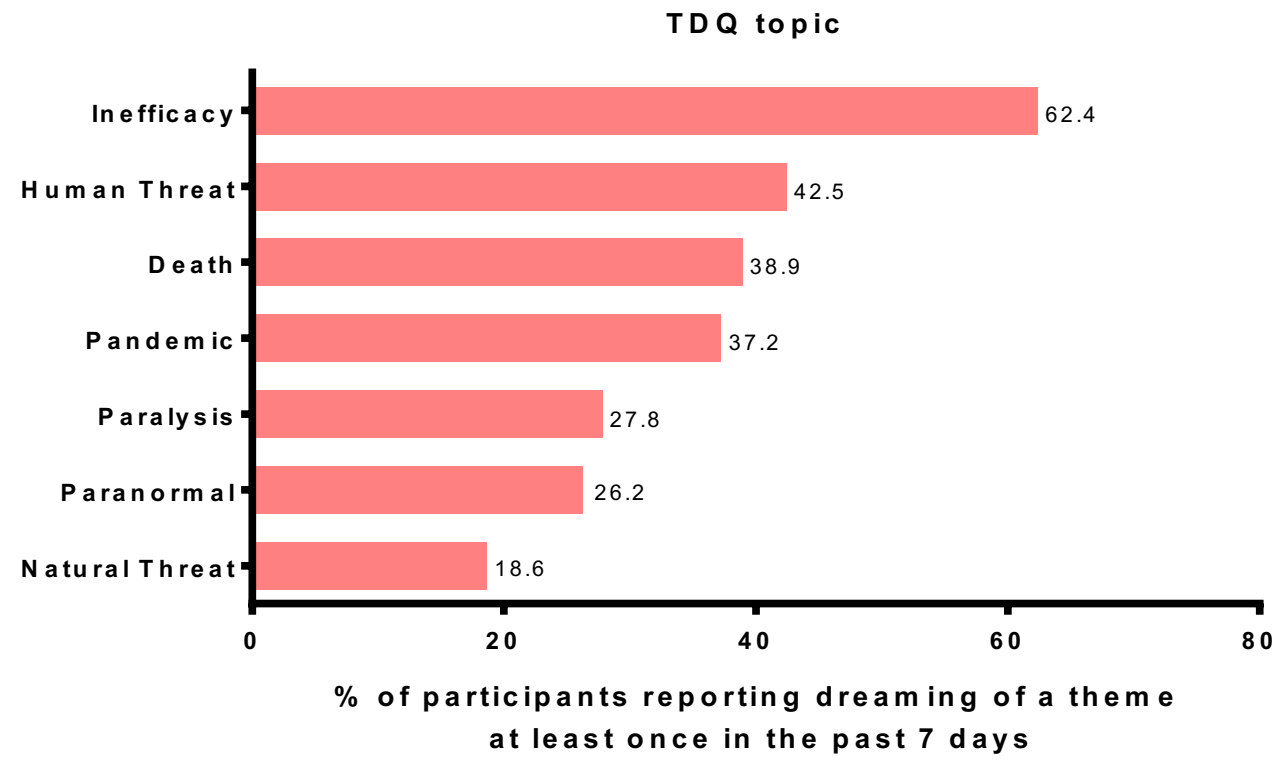

Figure 2. Frequency of the 7 dream topics 


\subsubsection{Dream recall, dream themes, stress and sleep}

A MANCOVA revealed a statistically significant difference between the stress level groups on the combined dependent variables $(F(6)=17.698, p<.001$; Wilks' $\Lambda=.894$; partial $\left.\eta^{2}=.055\right)$. Separate univariate follow-up ANOVAs with stress levels (low, moderate, severe) as independent factors and dream, bad dream and nightmare recall in the last 7 days, as well as sleep duration, as dependent factors, were performed. A separate ANOVA revealed that sleep duration decreased significantly as stress levels increased, and the frequency of all three dream recall variables increased as stress increased (Table 2).

Weekly dream recall: Tukey post-hoc comparisons revealed significant differences between low and severe stress groups (mean difference $=.762 ; p=.003$ ), and medium and severe stress groups (mean difference $=.579 ; p=.038$ ). No difference was observed between low and medium stress groups. Weekly bad dream recall: Tukey post-hoc comparisons revealed significant differences between all groups (low < moderate stress: mean difference $=.845$; moderate $<$ severe stress: mean difference $=1.11$; all $p<.001)$. Weekly nightmare recall: Tukey post-hoc comparisons revealed significant differences between all groups (low $<$ moderate stress: mean difference $=.367$; moderate $<$ severe stress: mean difference $=1.12$; all $\mathrm{p} \leq .001)$. Sleep duration: Tukey post-hoc comparisons revealed significant differences in hours spent asleep between low and severe stress groups (mean difference $=1.02, p<.001$ ), but not between low and moderate stress groups.

A MANCOVA revealed a statistically significant difference between the stress level groups on the combined dependent dream topic variables $(F(8)=7.021, p<.001$; Wilks' $\Lambda=$ .941 ; partial $\eta^{2}=.030$ ). Further, separate univariate ANCOVAs, with stress levels (low, moderate, severe) as independent factors, weekly dream recall as a covariate, and frequency of dreams associated with the four most prevalent dream topics (inefficacy, human threat, death and pandemic) as dependent measures, were performed (for means, standard deviations, $\mathrm{F}, p$, and $\eta_{\mathrm{p}}{ }^{2}$ values please see Table 2). Frequency of all dream themes significantly increased as stress levels increased (all $p<.001)$.

Results of these ANOVAs and ANCOVAs are also represented in Figure 2. 


\begin{tabular}{|c|c|c|c|c|c|c|c|}
\hline & $\begin{array}{l}\text { All } \\
\text { participants }\end{array}$ & Low stress & $\begin{array}{l}\text { Moderate } \\
\text { stress }\end{array}$ & $\begin{array}{l}\text { Severe } \\
\text { stress }\end{array}$ & $\mathbf{F}$ & $p$ & $\eta_{p}^{2}$ \\
\hline $\begin{array}{l}\mathbf{n} \\
\text { Weekly dream } \\
\text { recall }\end{array}$ & $\begin{array}{l}968 \\
(\mathbf{M} \pm \mathbf{S D})\end{array}$ & $\begin{array}{l}443 \\
(\mathbf{M} \pm \mathbf{S D})\end{array}$ & $\begin{array}{l}406 \\
(\mathbf{M} \pm \mathbf{S D})\end{array}$ & $\begin{array}{l}94 \\
(\mathbf{M} \pm \mathbf{S D})\end{array}$ & & & \\
\hline Dream recall & $2.9 \pm 2.1$ & $2.7 \pm 2.1$ & $2.9 \pm 2.0$ & $3.5 \pm 2.1$ & 5.36 & .005 & .01 \\
\hline Bad dream recall & $1.4 \pm 1.9$ & $0.8 \pm 1.4$ & $1.7 \pm 1.9$ & $2.8 \pm 2.5$ & 57.35 & $<001$ & .11 \\
\hline Nightmare & $0.8 \pm 1.5$ & $0.4 \pm 1.2$ & $0.8 \pm 1.3$ & $1.9 \pm 2.7$ & 41.96 & $<.001$ & .08 \\
\hline $\begin{array}{l}\text { Sleep duration } \\
\text { (hours per day) }\end{array}$ & $7.3 \pm 1.6$ & $7.5 \pm 0.1$ & $7.2 \pm 0.1$ & $6.4 \pm 0.2$ & 16.37 & $<.001$ & .03 \\
\hline \multicolumn{8}{|l|}{$\begin{array}{l}\text { Dream Topics } \\
\text { (TDQ) }\end{array}$} \\
\hline Pandemic & $4.9 \pm 1.7$ & $4.6 \pm 1.2$ & $5.0 \pm 1.7$ & $6.0 \pm 2.7$ & 26.38 & $<.001$ & .05 \\
\hline Death & $5.8 \pm 1.7$ & $5.6 \pm 1.3$ & $5.9 \pm 1.7$ & $6.7 \pm 2.6$ & 13.83 & $<.001$ & .03 \\
\hline Human Threat & $3.9 \pm 1.4$ & $3.6 \pm 1.1$ & $3.9 \pm 1.4$ & $4.8 \pm 2.3$ & 27.00 & $<.001$ & .06 \\
\hline Inefficacy & $6.7 \pm 2.2$ & $6.3 \pm 1.8$ & $6.9 \pm 2.2$ & $7.8 \pm 2.9$ & 17.84 & $<.001$ & .04 \\
\hline
\end{tabular}

Bonferroni correction: $\alpha=.05 / 8$ comparisons $=.006$

A

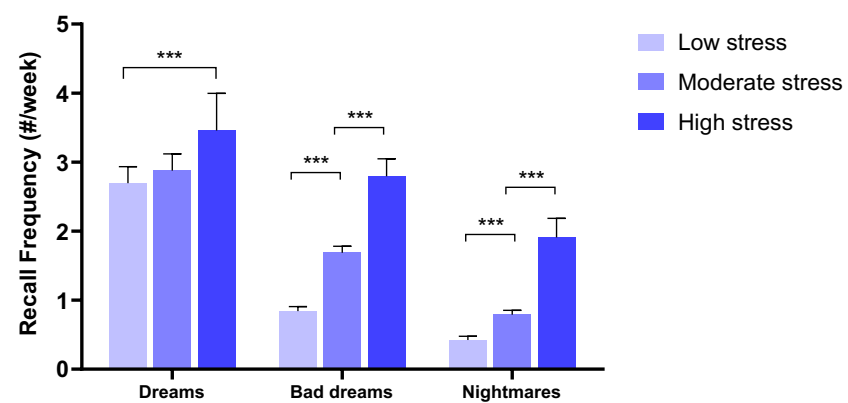

B

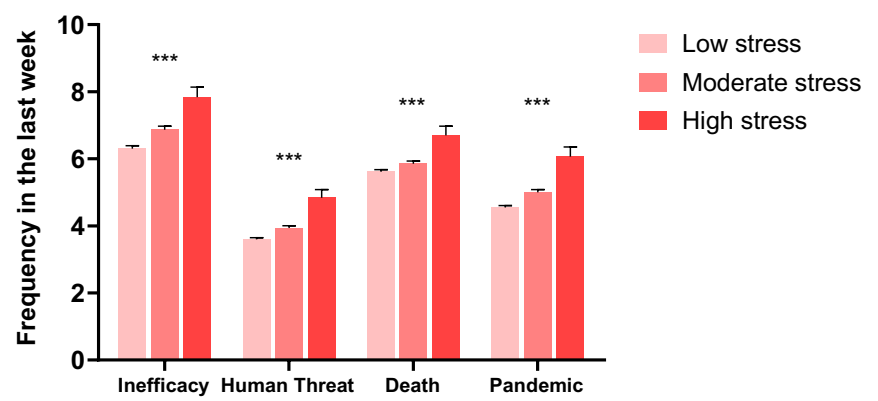

Figure 2. Comparison of low, moderate, and high stress groups on (A) dreams, bad dreams, and nightmares recall in the last 7 days, and on (B) the frequency of the top four dream topics (Inefficacy, Human Threat, Death and Pandemic) in the last 7 days. ${ }^{* * *} \mathrm{p}<.001$, Bonferroni-corrected for multiple comparisons.

\subsubsection{Relationship between dream recall, dream themes, stress, anxiety, depression and pandemic concerns}

Spearman correlation analyses between dream, bad dream and nightmare recall and measures of anxiety (GAD7), depression (QIDS) and stress (PSS) revealed positive relationships between all measures of dream recall and measures of psychopathology. The strongest 
correlations were weak and were found between frequency of bad dreams and stress, anxiety and depression symptoms. Dream recall was also associated with higher stress and anxiety, although these effects were small. The Spearman rho values and significance levels are reported in Table 3.

Table 3. Spearman correlations between dream, bad dream and nightmare recall, and anxiety, depression and stress symptoms

\begin{tabular}{llll}
\hline & PSS (stress) & QIDS (depression) & GAD7 (anxiety) \\
\hline dream recall & $.124^{*}$ & .155 & $.133^{*}$ \\
bad dreams recall & $.363^{*}$ & $.385^{*}$ & $.423^{*}$ \\
nightmares recall & .100 & .092 & .091 \\
\hline$*_{p}^{*}<001$ Bonferroni
\end{tabular}

${ }^{*} \mathrm{p}<.001$ Bonferroni correction: $\alpha=.05 / 9$ comparisons $=.005$

Spearman correlations between the frequency of the top four dream topics (inefficacy, human threat, death and pandemic imagery) and symptoms of anxiety (GAD7) and depression (QIDS), measures of stress (PSS), and concerns over the pandemic (whether the pandemic was a threat to their health, job or finances; and specific socio-medical concerns), were performed. All four dream topics were weakly positively correlated with stress, anxiety, and depression. Very weak positive associations were found between some of the frequencies of dream themes and pandemic concerns. For Spearman rhos and significance levels please see Table 4.

Table 4. Spearman correlation coefficients between dream topics and anxiety and depression symptoms, stress, perceptions of covid threat, and socio-medical concerns associated with COVID-19

\begin{tabular}{|c|c|c|c|c|}
\hline & Inefficacy & Human threat & Pandemic & Death \\
\hline \multicolumn{5}{|l|}{ Mental health } \\
\hline GAD7 (anxiety) & $.280 *$ & $.278 *$ & $.304 *$ & $.150 *$ \\
\hline QIDS (depression) & $.297 *$ & $.310 *$ & $.266^{*}$ & $.194 *$ \\
\hline PSS (stress) & $.234 *$ & $.235 *$ & $.261^{*}$ & $.125^{*}$ \\
\hline \multicolumn{5}{|l|}{ Covid Threats } \\
\hline health & ns & ns & ns & ns \\
\hline job & ns & ns & ns & ns \\
\hline finances & ns & $-113 *$ & ns & -.101 \\
\hline \multicolumn{5}{|l|}{ Covid concerns } \\
\hline lacking food & .077 & $.142 *$ & $.122 *$ & ns \\
\hline public services shutting down & .095 & $.147 *$ & $.179 *$ & .092 \\
\hline schools closed & .086 & ns & .106 & .086 \\
\hline children or relatives not coping well & $.155^{*}$ & $.154 *$ & .164 & ns \\
\hline not being able to access medical services & $.147 *$ & $.155^{*}$ & $.181 *$ & $.135^{*}$ \\
\hline
\end{tabular}

$* \mathrm{p}<.001$ Bonferroni correction: $\alpha=.05 / 44$ comparisons $=.001$

\subsubsection{Linear regression analyses}


Stress levels: Multiple linear regression model statistically significantly predicted PSS scores $F(12,748)=29.248, p<.001$, adj. $R^{2}=.32$. Among dream variables, nightmare and bad dream frequency (both $\mathrm{p}<.001$ ) and pandemic dream topic significantly predicted PSS scores $(p=.037)$. The death topic showed a non-significant trend $(p=.075)$. In addition, younger age $(\mathrm{p}<.001)$, female sex $(\mathrm{p}=.013)$ and antidepressant use $(\mathrm{p}=.001)$ predicted PSS scores.

Depression: Multiple linear regression model statistically significantly predicted QIDS scores $F(12,751)=32.354, p<.001$, adj. $R^{2}=.33$. Among dream variables, nightmare and bad dream frequency (both $\mathrm{p}<.001)$ and death dream topic $(\mathrm{p}<.001)$, and inefficacy dream topic $(p=.032)$ significantly predicted QIDS scores. In addition, younger age $(p<.001)$, lower income status $(\mathrm{p}=.037)$ and antidepressant use $(\mathrm{p}<.001)$ predicted QIDS scores.

Anxiety: Multiple linear regression model statistically significantly predicted GAD7 scores $F(12,779)=32.127, p<.001$, adj. $R^{2}=.33$. Among dream variables, nightmare and bad dream frequency (both $\mathrm{p}<.001)$, dream recall frequency $(\mathrm{p}=.010)$, pandemic dream topic $(\mathrm{p}<.001)$ and death dream topic $(\mathrm{p}<.029)$, significantly predicted GAD7 scores. In addition, younger age $(p<.001)$, female sex $(p=.018)$, and antidepressant use $(p<.031)$ predicted GAD7 scores. These results are presented in Table 5.

Table 5. Multiple regression results for PSS, QIDS and GAD7 scores

\begin{tabular}{|c|c|c|c|c|c|c|c|}
\hline & \multirow[t]{2}{*}{$\boldsymbol{B}$} & \multicolumn{2}{|c|}{$95 \%$ CI for $B$} & \multirow[t]{2}{*}{$S E B$} & \multirow[t]{2}{*}{$\beta$} & \multirow[t]{2}{*}{$R^{2}$} & \multirow[t]{2}{*}{$\Delta \mathbf{R}^{2}$} \\
\hline & & $L L$ & $\boldsymbol{U L}$ & & & & \\
\hline \multicolumn{8}{|l|}{ PSS } \\
\hline Model & & & & & & .319 & $.308 * * *$ \\
\hline Constant & $21.499 * * *$ & 19.540 & 23.457 & .998 & & & \\
\hline $\begin{array}{l}\text { Nightmare } \\
\text { frequency }\end{array}$ & $.755^{* * *}$ & .356 & 1.154 & .203 & $.138 * * *$ & & \\
\hline $\begin{array}{l}\text { Bad dream } \\
\text { frequency }\end{array}$ & $.592 * * *$ & .260 & .923 & .169 & $.130 * * *$ & & \\
\hline Dream recall & -.214 & .066 & .775 & .142 & -.051 & & \\
\hline $\begin{array}{l}\text { Pandemic } \\
\text { dream topic }\end{array}$ & $.596^{*}$ & .036 & 1.156 & .285 & $.073 *$ & & \\
\hline $\begin{array}{l}\text { Death dream } \\
\text { topic }\end{array}$ & .609 & -.060 & 1.279 & .341 & .067 & & \\
\hline $\begin{array}{l}\text { Inefficacy } \\
\text { dream topic }\end{array}$ & .394 & -.113 & .902 . & .258 & .055 & & \\
\hline $\begin{array}{l}\text { Human threat } \\
\text { dream topic }\end{array}$ & .077 & -.687 & .841 & .389 & .008 & & \\
\hline Age & $-.163 * * *$ & -.194 & -.131 & .016 & $-.333 * * *$ & & \\
\hline Sex & $-1.442 *$ & -2.575 & -.309 & .577 & $-.079 *$ & & \\
\hline Income status & 1.095 & -.498 & 2.668 & .811 & .042 & & \\
\hline $\begin{array}{l}\text { Employment } \\
\text { status }\end{array}$ & -.024 & -.076 & .027 & .026 & .138 & & \\
\hline
\end{tabular}




\begin{tabular}{|c|c|c|c|c|c|c|c|}
\hline \multirow{3}{*}{$\begin{array}{l}\text { Antidepressant } \\
\text { use }\end{array}$} & $1.954 * * *$ & .826 & 3.083 & .575 & $.108 * * *$ & & \\
\hline & \multirow[t]{2}{*}{$B$} & \multicolumn{2}{|c|}{$95 \%$ CI for $B$} & \multirow[t]{2}{*}{$S E B$} & \multirow[t]{2}{*}{$\beta$} & \multirow[t]{2}{*}{$R^{2}$} & \multirow[t]{2}{*}{$\Delta \mathbf{R}^{2}$} \\
\hline & & $L L$ & $U \boldsymbol{U}$ & & & & \\
\hline \multicolumn{8}{|l|}{ QIDS } \\
\hline Model & & & & & & .341 & $.330 * * *$ \\
\hline Constant & $10.429 * * *$ & 9.214 & 11.644 & .619 & & & \\
\hline $\begin{array}{l}\text { Nightmare } \\
\text { frequency }\end{array}$ & $.537 * * *$ & .288 & .786 & .127 & $.155^{* * *}$ & & \\
\hline $\begin{array}{l}\text { Bad dream } \\
\text { frequency }\end{array}$ & $.403 * * *$ & .196 & .610 & .105 & $.140 * * *$ & & \\
\hline Dream recall & -.104 & -.278 & .071 & .089 & -.039 & & \\
\hline $\begin{array}{l}\text { Pandemic } \\
\text { dream topic }\end{array}$ & .119 & -.232 & .470 & .179 & .023 & & \\
\hline $\begin{array}{l}\text { Death dream } \\
\text { topic }\end{array}$ & $.857 * * *$ & .440 & 1.275 & .213 & $.148 * * *$ & & \\
\hline $\begin{array}{l}\text { Inefficacy } \\
\text { dream topic }\end{array}$ & $.346^{*}$ & .031 & .662 & .161 & $.076^{*}$ & & \\
\hline $\begin{array}{l}\text { Human threat } \\
\text { dream topic }\end{array}$ & .259 & -.216 & .733 & .242 & .040 & & \\
\hline Age & $-.074 * * *$ & -.093 & -.054 & .010 & $-.238 * * *$ & & \\
\hline Sex & -.700 & -1.406 & .006 & .360 & -.061 & & \\
\hline Income status & $1.044^{*}$ & .061 & 2.028 & .501 & $.063^{*}$ & & \\
\hline $\begin{array}{l}\text { Employment } \\
\text { status }\end{array}$ & -.020 & -.052 & .012 & .016 & -.036 & & \\
\hline \multirow{3}{*}{$\begin{array}{l}\text { Antidepressant } \\
\text { use }\end{array}$} & $1.692 * * *$ & .989 & 2.394 & .358 & $.148 * * *$ & & \\
\hline & B & \multicolumn{2}{|c|}{$95 \%$ CI for $B$} & $S E B$ & $\boldsymbol{\beta}$ & $R^{2}$ & $\Delta \mathbf{R}^{2}$ \\
\hline & & $L L$ & $\boldsymbol{U L}$ & & & & \\
\hline \multicolumn{8}{|l|}{ GAD7 } \\
\hline Model & & & & & & .340 & $.329 * * *$ \\
\hline Constant & $9.124 * * *$ & 7.810 & 10.437 & .669 & & & \\
\hline $\begin{array}{l}\text { Nightmare } \\
\text { frequency }\end{array}$ & $.686^{* * *}$ & .418 & .957 & .137 & $.184 * * *$ & & \\
\hline $\begin{array}{l}\text { Bad dream } \\
\text { frequency }\end{array}$ & $.525 * * *$ & .303 & .748 & .113 & $.169 * * *$ & & \\
\hline Dream recall & $-.246 * *$ & -.434 & -.058 & .096 & -.087 & & \\
\hline $\begin{array}{l}\text { Pandemic } \\
\text { dream topic }\end{array}$ & $.683 * * *$ & .308 & 1.058 & .191 & $.124 * * *$ & & \\
\hline $\begin{array}{l}\text { Death dream } \\
\text { topic }\end{array}$ & $.501 *$ & .051 & .950 & .229 & $.080 *$ & & \\
\hline $\begin{array}{l}\text { Inefficacy } \\
\text { dream topic }\end{array}$ & .261 & -.079 & .601 & .173 & .053 & & \\
\hline $\begin{array}{l}\text { Human threat } \\
\text { dream topic }\end{array}$ & .236 & -.275 & .748 & .261 & .034 & & \\
\hline Age & $-.089 * * *$ & -.110 & -.068 & .011 & $-.267 * * *$ & & \\
\hline Sex & $-.915^{*}$ & -1.675 & -.155 & .387 & $-.074^{*}$ & & \\
\hline Income status & .014 & -1.044 & 1.073 & .539 & .001 & & \\
\hline $\begin{array}{l}\text { Employment } \\
\text { status }\end{array}$ & .028 & -.062 & .007 & .018 & -.048 & & \\
\hline $\begin{array}{l}\text { Antidepressant } \\
\text { use }\end{array}$ & $.835^{*}$ & .076 & 1.594 & .387 & $.068^{*}$ & & \\
\hline
\end{tabular}


Model= "Enter" method in SPSS Statistics; $B=$ unstandardized coefficient; $\mathrm{CI}=$ confidence interval; $\mathrm{LL}=$ lower limit; $\mathrm{UL}=$ upper limit; SE $B=$ standard error of the coefficient; $\beta=$ standardized coefficient; $R^{2}=$ coefficient of determination; $\Delta R^{2}=$ adjusted $R^{2} .{ }^{*} p<.05 ; * * p .01 ; * * * p<.001$. Sex binary measure: $0=$ female, $1=$ male.

\section{Discussion}

During the acute phase of the first COVID-19 lockdown (April 3-May 15, 2020), over half of the respondents to our national longitudinal online survey reported a change in habitual patterns of dream recall (34\% reported increased and 19\% reported decreased dream recall). The most prevalent dream themes centered around topics of inefficacy, threat from other humans, death, and pandemic imagery. Pandemic-related dreams were reported by $37 \%$ of our participants, while dreams characterized by inefficacy were reported by $62 \%$ of participants, with the most prevalent individual theme being trying again and again to do something (51\%). The frequency of dream, bad dream and nightmare recall was associated with concomitant levels of stress, with higher frequency of recall in higher stress groups. Similarly, frequency of dreams centered around the four most prevalent dream topics (inefficacy, human threat, death and pandemic imagery) was also associated with higher levels of stress. Lastly, bad dream recall and the four most prevalent dream topics were all also positively correlated with symptoms of anxiety and depression.

\subsection{Dream, bad dream and nightmare recall.}

In our sample, the majority of participants reported a change in dream recall during the pandemic lockdown (last 7 days) in comparison to their retrospective estimate (last year). Over a third (34\%) of participants reported an increase in dream recall, while almost a fifth of participants (20\%) reported a decrease, and about half (46\%) showed no change in dream recall. This pattern is somewhat consistent with findings of a survey study by Schredl and Bulkeley (2020): $29 \%$ of participants of a US-based survey $(n=3,031)$ stated that they remembered more dreams than before the pandemic. In contrast, in our sample, we report a higher $(19 \% \mathrm{vs} .7 \%$ in Schredl \& Bulkeley study) proportion of participants whose dream recall has decreased. This discrepancy is potentially due to differences in methodology: we compared retrospective estimates of dream recall (past year and past 7 days) to determine whether dream recall has changed, while in Schredl \& Bulkeley's survey, participants were asked to rate whether their 
dream recall decreased, stayed the same or increased. Contrary to our expectations, and the existing literature, in our sample, overall bad dream and nightmare recall did not increase during the lockdown, as compared to the past year.

Further, and contrary to the sleep extension hypothesis of increased dreaming during the pandemic (Bottary, Simonelli, Cunningham, Kensinger, \& Mantua, 2020), we did not find an association between dream recall and sleep duration. We did, however, find weak negative correlations between sleep duration and bad dream and nightmare recall, which suggests that shorter sleep time in our sample was associated with higher rates of dysphoric dreaming specifically. This finding is consistent with a recent study in Chinese adolescents showing an association between short sleep time and recurrent nightmares (Lin et al., 2020). However, other studies either report a positive relationship between nightmare frequency and longer sleep duration (Rek, Sheaves, \& Freeman, 2017; Sandman et al., 2013) or no association between nightmares and sleep duration (Nielsen, 2010). It is possible, nevertheless, that a combination of sleep disturbances during the lockdown, such as shorter sleep duration, has a different relationship to disturbed dreaming than habitual patterns of shorter or longer sleep time. We previously observed three general patterns of sleep change since the pandemic (Robillard, Dion, et al., 2021), identifying three clusters: reduced time in bed, delayed sleep and extended time in bed. The two first groups showed higher rates of symptoms of stress, anxiety and depression than the latter group, suggesting that sleep extension potentially had a buffering effect on the experience of lockdown challenges, while reduced sleep time potentially represented maladaptive behavioural and psychological responses to lockdown.

\subsection{Dream themes}

Overall, the most common dream themes corresponded relatively well to existing prepandemic literature. However, some changes in the prevalence of themes were also observed during lockdown. Strikingly, the most common dream theme was "trying over and over again to do something", and it significantly surpassed the "being chased or pursued" dream theme which was most commonly reported before the pandemic in the Canadian sample (Nielsen et al., 2003), second most prevalent in the German (Schredl et al., 2004) and Chinese (Yu, 2008) samples, after "school, teachers and studying" in both latter cases. In addition, the new theme, "being separated from a loved one" was also highly prevalent. These results suggest that typical dream 
themes appear to be relatively stable (being chased, sexual experiences, school, falling, arriving late, etc.) despite potentially important changes in organization of individual lives (in this case, during the pandemic-related lockdown). Second, the specific themes that become more pronounced during particular individual and collective challenges, reflect, broadly, the ongoing situation. In our case, items such as "trying" and "being separated from a loved one" were some of the most prominent, representing global psychological concerns during this time.

Dream themes centered around specific elements associated with the pandemic, with the exception of "being separated from a loved one" (hospitals, being sick, germs and contamination) were not very prevalent individually (7.64\% for germs/contamination; $12 \%$ for being in a hospital; and $14.8 \%$ for being sick), and when put together represented the fourth most prevalent topic, after inefficacy, human threat and death imagery. This is consistent with the idea that the dreaming mind, instead of incorporating episodic memories directly and explicitly into oneiric content, extracts the gist of the experience. Indeed, the COVID-19 pandemic (as opposed, for example, to other important individual and collective events), during the first lockdown, was not characterized by particularly recognizable or striking imagery. With the exception of face coverings (which were not mandatory in Canada during the lockdown in April and May 2020), for the majority of respondents, who were not considered essential workers, the pandemic played out mostly outside of the walls of their houses. Considering this, it is perhaps not surprising that the most common dream themes were associated with an overarching topic of inefficacy. Dream themes associated with the topics of inefficacy, human threat, and increased bad dreams and nightmares in medium and high stress groups, also potentially reflected the psychosocial and affective challenges that many faced during the lockdown. Studies from numerous countries report elevated rates of distressing experiences and emotions associated with the emerging pandemic (Khan, Mamun, Griffiths, \& Ullah, 2020). Fear in general has been reported by multiple teams (Ahorsu et al., 2020; Fitzpatrick, Harris, \& Drawve, 2020; Trnka \& Lorencova, 2020). Among other emotional responses found in the current literature, which may be congruent with the most common dream themes, were anger, hopelessness (Trnka \& Lorencova, 2020), and loneliness (Groarke et al., 2020).

The fact that pandemic imagery was not reported by the majority of participants, despite its salient nature, is consistent with the rates of $8 \%$ the pandemic-related dreams reported in a U.S.-based survey (Schredl \& Bulkeley, 2020), and with the Italian study (Iorio et al., 2020) that 
reports that $20 \%$ of their participants had a dream about the pandemic. This finding is also consistent, in a more general way, with studies of stimuli incorporation into dreams. For instance, a study tracking students' dreams during the exam period (Delorme, Lortie-Lussier, \& De Koninck, 2002) did not reveal an increase of dreams about exams. The mechanisms of stimuli incorporation and functions of memory sources in dreams are still unclear. Indeed, the belief update mechanisms by which (and speed at which) environmentally and culturally novel information can be integrated and automatized remain poorly understood (Strößner, 2020).

In fact, while elements of lived experience (memories, thoughts, emotions) make up dream content, full episodic memory incorporations are virtually absent in healthy individuals (Fosse, Fosse, Hobson, \& Stickgold, 2003; Nielsen \& Stenstrom, 2005). Rather, dreams integrate memories in an associative way, bringing together elements of temporally and contextually different episodic memories to create novel scenarios (Horton \& Malinowski, 2015; Stenstrom, Fox, Solomonova, \& Nielsen, 2012). One of the proposed potential functions of dreams is that the dreaming brain extracts the gist of autobiographically salient experiences (Blagrove et al., 2014; Eichenlaub et al., 2017; Malinowski \& Horton, 2014; Wamsley \& Stickgold, 2011). In accordance with this view, we propose that the increase in inefficacy dreams reported in our sample reflected the specific aspects of quality of diminished life opportunities and life plans put on hold under the lockdown. Similarly, high prevalence of dreams of "being separated from a loved one" found in our sample, reinforces the notion that dreams preferentially incorporate elements of waking life that are personally significant and reflect our species' inextricably social, relational, and affective nature (Veissiere, Constant, Ramstead, Friston, \& Kirmayer, 2019). Absence of loved ones may not be directly attributed to the pandemic imagery in itself, but social deprivation during lockdown is certainly characterized by very marked lack of opportunity to spend time with others. Overall, it is likely that environmentally novel information related to the COVID-19 pandemic would not have been sufficiently entrained to be automatized into dream content (the way one would not in a foreign language through mere travelling exposure), whereas evolutionarily invariant attentional biases toward threat- and infection-detection, and maintaining social bonds and obligations (Veissière et al, 2020) would flare-up in times of increased uncertainty. 


\subsection{Dreams, nightmares, stress and symptoms of depression and anxiety}

Contrary to other studies (Bruni et al., 2021; Giardino et al., 2020; Kilius et al., 2021; Pesonen et al., 2020; Tu et al., 2020), we did not observe an overall increase in bad dream or nightmare frequency during the lockdown, as compared to the pre-lockdown subjective estimate. This is nonetheless consistent with the current research suggesting that propensity towards nontraumatic nightmares may be a relatively stable trait, reflecting a global tendency towards affect distress (Levin \& Nielsen, 2007), which potentially develops from early experiences with adversity (Nielsen, 2017) and alters both the overall affect regulation pathways (Gieselmann et al., 2019; Nielsen \& Levin, 2007) and cognitive appraisal of the experience of nightmares (Gieselmann, Elberich, Mathes, \& Pietrowsky, 2020). Both nightmares (Carr \& Nielsen, 2017) and sleep quality in general (Drake, Pillai, \& Roth, 2014) have been proposed to be representative of stress reactivity. This reactivity may be a trait, which, according to stressdiathesis and differential susceptibility theories (Belsky \& Pluess, 2009; Monroe \& Simons, 1991), predisposes an individual towards a stronger response to environmental stressors. Thus, increased nightmare and bad dream frequency in individuals suffering from moderate to severe stress response during the lockdown may indicate a cross-state reactivity phenomenon.

Our team has previously reported that shorter sleep duration was associated with increased stress levels in the same sample (Robillard, Saad, et al., 2020). In the present study we did not find an association between sleep duration and dream recall, with the exception for bad dreams, which suggests that dream recall in general may be independently associated with stress, and thus may represent an indirect marker of stress reactivity/psychological distress.

Further, weak positive associations/trends were found for the relationships between the four most prevalent dream themes (inefficacy, human threat, pandemic and death) and concerns over COVID-19-related social changes (lacking food, public services shutting down, school closures, children or relatives not coping well, and not being able to access medical services).

Lastly, in addition to the well-established notion that dysphoric dreaming reacts to stressors and is affected by psychopathology, an emerging body of research suggests that bad dreams and nightmares may exacerbate or even trigger increased stress response (Garcia et al., 2021), contribute to daytime distress (Gehrman, Harb, Cook, Barilla, \& Ross, 2015; Gieselmann et al., 2020; Levin \& Fireman, 2002) and symptoms of psychopathology, including anxiety (Roberts, Lennings, \& Heard, 2009) and depression (Hedstrom et al., 2021). Thus, to account for 
the possibility of the bi-directional relationship between dreams, stress and psychopathology, we tested whether dream, bad dream and nightmare frequency, and the most frequent dream topics predicted levels of stress and symptoms of depression and anxiety. Bad dreams and nightmares predicted stress, depression and anxiety in our sample; pandemic-themed dreams predicted levels of stress; inefficacy dreams predicted symptoms of depression, and death dreams predicted both depression and anxiety symptoms. In addition, female gender predicted stress and anxiety levels, and younger age and antidepressant use predicted all three outcome variables. These results indicate a potential role that bad dreams and nightmares may play in amplifying the experience of stress, depression and anxiety.

Further, different dream topics were associated with different mental health outcomes. Pandemic-themed dream frequency was associated only with stress, suggesting that offline preoccupation with the ongoing health crisis can be seen as a feedback loop factor which is both caused by stress associated with the pandemic and, in turn, may increase stress level. This is consistent with general theories of nightmares and affect distress (Carr \& Nielsen, 2017; Nielsen \& Lara-Carrasco, 2007; Nielsen \& Levin, 2007; Nielsen \& Carr, 2017), lending further support to the notion that a cross-state environmental reactivity or susceptibility trait may serve as a predisposing factor for distress response in face of a challenging situation.

Inefficacy dreams, the most common dream topic in our sample, predicted only symptoms of depression and not stress or anxiety. The topic of inefficacy is characterized by themes of not being able to do what one needs or wants to, not being able to accomplish one's goals. All these themes can be related to some of the prominent characteristics of depressive symptoms, such as helplessness (Pryce et al., 2011), a tendency towards external locus of control (Hovenkamp-Hermelink et al., 2019), and low self-efficacy (Rosas et al., 2019).

Death dreams, characterized by themes such as seeing someone alive as dead, encountering people who are dead, dying or killing, on the other hand, predicted both depression and anxiety. The topic of death dreams is related to the most salient fears in context of the pandemic. Death anxiety has been previously associated with depression (Iverach, Menzies, \& Menzies, 2014), and more recently, a novel concept of coronaphobia (Asmundson \& Taylor, 2020) was proposed to describe distressing psychological symptoms, including anxiety and depression, associated specifically with fears related to COVID-19. In a recent study, coronaphobia was positively associated with, depression, anxiety and death anxiety (Lee, Jobe, 
Mathis, \& Gibbons, 2020). Consistent with literature on the relationship between current preoccupations and dream content, these results indicate an affective cross-state continuity between daytime and nighttime experiences, and suggest a potential role for dreams in emotion regulation and in accentuating symptoms of psychopathology.

\subsection{Methodological issues and limitations}

One of the main limitations of the present study is the self-selected nature of our participants. The TDQ was part of the optional component of the "How are you coping" survey: participants first filled out the main questionnaire and then were asked if they wanted to answer more questions. Thus, our sample consisted of motivated and interested participants, potentially limiting generalizability to the general population affecting generalizability. In addition to the selection bias, our sample potentially is characterized by retrospective reporting bias (estimates over the last year, vs. the last week). Since we are sampling both time points (before and during pandemic) at the same time, our study is not truly of longitudinal nature. One of the main strengths of this study, however, is the fact that because the TDQ was part of the optional and additional component of the study, our participants did not specifically expect to be asked questions about their dreams. In fact, the survey was advertised as a project on mental health and socio-economic impact of the pandemic. This aspect of our study differentiates our survey from the vast majority of dream research projects, where participants are explicitly recruited to take part in a study on dreams and are potentially self-selected on the basis of their pre-existing interest in dreams. Thus, our sample potentially provides a more generalizable view of oneiric lives during the lockdown.

Further, the pandemic-related themes that we added to the TDQ following the announcement of the first lockdown in March 2020 do not cover the full range of pandemic-related imagery that became salient parts of our lives under the "new normal" as the pandemic progressed (themes such as social distancing, dystopic futures, etc. that were recently identified as common pandemic-related imagery (e.g. Pesonen et al, 2020). In addition, the TDQ was originally used to identify dream themes over a lifetime, and we assessed them over a much more limited span of the last 7 days, to sample the most recent dream themes occurring specifically in the context of the lockdown. Therefore, the comparisons with the previous literature have to be nuanced, bearing in mind the temporal scale differences between our study and earlier research. 


\section{Conclusions}

We report changes in dream content during the first COVID-19 lockdown (April-May 2020), and a relationship between dreams, bad dreams, nightmares, and dream themes to stress, depression and anxiety. Dream recall, bad dream and nightmare frequency increased during the lockdown, particularly for individuals with moderate to high stress, potentially representing individual tendency towards environmental susceptibility and global traits for affect distress and stress reactivity. The most prevalent dream themes were centered around topics of inefficacy, human threat, pandemic and death imagery. The frequency of these dreams was positively associated with stress levels, anxiety and depression symptomatology. Lastly, bad dreams and nightmares were associated with symptoms of stress, depression and anxiety, and different dream topics predicted different mental health outcomes. Specifically, pandemic dreams were associated with stress, inefficacy dreams were associated with depression, and death dreams were associated with both depression and anxiety. We suggest that the unique lived experience of life under a pandemic lockdown produced changes not only in general mental health and sleep patterns, but also in oneiric life, reflecting in a general sense, the ongoing existential challenges. Specifically, most common dream themes during this time centered around the topic of inefficacy (trying over and over again, arriving too late, etc.), and dreams of being separated from loved ones evoke the overall feeling of lives put on hold, and possibly represent the process of making sense of this new experience. These results are consistent with theories emphasizing the associative nature of memory processing in dreams and the role of emotionally charged and personally significant events in dream formation.

\section{Financial Disclosure Statement}

The authors received no specific funding for this work. ES was supported by a Fonds de Recherche du Québec - Société et Culture (FRQSC) postdoctoral fellowship; MP was supported by a grant from Fonds de Rercherche du Québec - Santé. 


\section{References}

Ahorsu, D. K., Lin, C. Y., Imani, V., Saffari, M., Griffiths, M. D., \& Pakpour, A. H. (2020). The Fear of COVID-19 Scale: Development and Initial Validation. Int J Ment Health Addict, 1-9. doi:10.1007/s11469-020-00270-8

Alvaro, P. K., Roberts, R. M., \& Harris, J. K. (2013). A Systematic Review Assessing Bidirectionality between Sleep Disturbances, Anxiety, and Depression. Sleep, 36(7), 1059-1068. doi:10.5665/sleep.2810

Asmundson, G. J. G., \& Taylor, S. (2020). Coronaphobia: Fear and the 2019-nCoV outbreak. $J$ Anxiety Disord, 70, 102196. doi:10.1016/j.janxdis.2020.102196

Barrett, D. (2020). Dreams About COVID-19 Versus Normative Dreams: Trends by Gender. Dreaming, 30(3), 216-221.

Barrett, D., Sogolow, Z., Angela, O. H., Panton, J., Grayson, M., \& Justiniano, M. (2013). Content of dreams from WWII POWs. Imagination, Cognition and Personality, 33(1), 193-204.

Belsky, J., \& Pluess, M. (2009). Beyond diathesis stress: differential susceptibility to environmental influences. Psychol Bull, 135(6), 885-908. doi:10.1037/a0017376

Bergman, M., MacGregor, O., Olkoniemi, H., Owkzarski, W., Revonsuo, A., \& Valli, K. (2020). The Holocaust as a Lifelong Nightmare: Posttraumatic Symptoms and Dream Content in Polish Auschwitz Survivors 30 Years After World War II. The American Journal of Psychology, 133(2), 143-166.

Blagrove, M., Eichenlaub, J. B., van Rijn, E., Phelan, M., Ryder, L., Gaskell, G., .. . Walker, M. (2014). The nature of delayed dream incorporation: Personally significant events persist, but not major daily activities or concerns. Paper presented at the 22nd Congres of European Sleep Research Society, Tallinn, Estonia.

Blume, C., Schmidt, M. H., \& Cajochen, C. (2020). Effects of the COVID-19 lockdown on human sleep and rest-activity rhythms. Current Biology, 30(14), R795-R797. doi:10.1016/j.cub.2020.06.021

Bottary, R., Simonelli, G., Cunningham, T. J., Kensinger, E. A., \& Mantua, J. (2020). Sleep extension: an explanation for increased pandemic dream recall? Sleep. doi:10.1093/sleep/zsaa131

Bruni, O., Malorgio, E., Doria, M., Finotti, E., Spruyt, K., Melegari, M. G., . . Ferri, R. (2021). Changes in sleep patterns and disturbances in children and adolescents in Italy during the Covid-19 outbreak. Sleep Med. doi:10.1016/j.sleep.2021.02.003

Bulkeley, K., \& Kahan, T. L. (2008). The impact of September 11 on dreaming. Consciousness and Cognition, 17(4), 1248-1256. doi:10.1016/j.concog.2008.07.001

Buysse, D. J., Reynolds, C. F., 3rd, Monk, T. H., Berman, S. R., \& Kupfer, D. J. (1989). The Pittsburgh Sleep Quality Index: a new instrument for psychiatric practice and research. Psychiatry Res, 28(2), 193-213. Retrieved from https://www.ncbi.nlm.nih.gov/pubmed/2748771

Carey, B. (2020). What We Dream When We Dream About COVID-19. The New York Times. Retrieved from https:/www.nytimes.com/2020/10/06/health/dreams-covidcoronavirus.html

Carr, M., \& Nielsen, T. (2017). A novel Differential Susceptibility framework for the study of nightmares: Evidence for trait sensory processing sensitivity. Clin Psychol Rev, 58, 8696. doi:10.1016/j.cpr.2017.10.002 
Casagrande, M., Favieri, F., Tambelli, R., \& Forte, G. (2020). The enemy who sealed the world: effects quarantine due to the COVID-19 on sleep quality, anxiety, and psychological distress in the Italian population. Sleep Med, 75, 12-20. doi:10.1016/j.sleep.2020.05.011

Cellini, N., Canale, N., Mioni, G., \& Costa, S. (2020). Changes in sleep pattern, sense of time and digital media use during COVID-19 lockdown in Italy. Journal of Sleep Research, e13074. doi:10.1111/jsr.13074

Cohen, S., Kamarck, T., \& Mermelstein, R. (1983). A global measure of perceived stress. Journal of Health and Social Behavior, 24(4), 385-396. Retrieved from https://www.ncbi.nlm.nih.gov/pubmed/6668417

Collie, M. (2020, April 14, 2020). Having weird dreams during the coronavirus outbreak? You're not alone. Global News. Retrieved from [https://globalnews.ca/news/6814991/coronavirus-dreams

Dal Santo, F., Gonzalez-Blanco, L., Rodriguez-Revuelta, J., Marina Gonzalez, P. A., Paniagua, G., Garcia-Alvarez, L., . . Bobes, J. (2021). Early Impact of the COVID-19 Outbreak on Sleep in a Large Spanish Sample. Behav Sleep Med, 1-16. doi:10.1080/15402002.2021.1890597

Delorme, M. A., Lortie-Lussier, M., \& De Koninck, J. (2002). Stress and Coping in the Waking and Dreaming States During an Examination Period. Dreaming, 12(4), 171-183.

Drake, C. L., Pillai, V., \& Roth, T. (2014). Stress and sleep reactivity: a prospective investigation of the stress-diathesis model of insomnia. Sleep, 37(8), 1295-1304. doi:10.5665/sleep.3916

Eichenlaub, J. B., Cash, S., \& Blagrove, M. (2017). Daily Life Experiences in Dreams and Sleep-Dependent Memory Consolidation. In N. Axmacher \& B. Rasch (Eds.), Cognitive Neuroscience of Memory Consolidation. Switzerland: Springer Nature Switzerland AG.

Fitzpatrick, K. M., Harris, C., \& Drawve, G. (2020). Fear of COVID-19 and the mental health consequences in America. Psychol Trauma, 12(S1), S17-S21. doi:10.1037/tra0000924

Fosse, M. J., Fosse, R., Hobson, J. A., \& Stickgold, R. J. (2003). Dreaming and episodic memory: a functional dissociation? J Cogn Neurosci, 15(1), 1-9. doi:10.1162/089892903321107774

Garcia, O., Slavish, D. C., Dietch, J. R., Messman, B. A., Contractor, A. A., Haynes, P. L., . . Taylor, D. J. (2021). What goes around comes around: Nightmares and daily stress are bidirectionally associated in nurses. Stress Health. doi:10.1002/smi.3048

Gasteiger, N., Vedhara, K., Massey, A., Jia, R., Ayling, K., Chalder, T., . . . Broadbent, E. (2021). Depression, anxiety and stress during the COVID-19 pandemic: results from a New Zealand cohort study on mental well-being. BMJ Open, 11(5), e045325. doi:10.1136/bmjopen-2020-045325

Gehrman, P. R., Harb, G. C., Cook, J. M., Barilla, H., \& Ross, R. J. (2015). Sleep diaries of Vietnam War veterans with chronic PTSD: the relationships among insomnia symptoms, psychosocial stress, and nightmares. Behav Sleep Med, 13(3), 255-264. doi:10.1080/15402002.2014.880344

Giardino, D. L., Huck-Iriart, C., Riddick, M., \& Garay, A. (2020). The endless quarantine: the impact of the COVID-19 outbreak on healthcare workers after three months of mandatory social isolation in Argentina. Sleep Med, 76, 16-25. doi:10.1016/j.sleep.2020.09.022

Gieselmann, A., Ait Aoudia, M., Carr, M., Germain, A., Gorzka, R., Holzinger, B., . . . Pietrowsky, R. (2019). Aetiology and treatment of nightmare disorder: State of the art and future perspectives. Journal of Sleep Research, 28(4), e12820. doi:10.1111/jsr.12820 
Gieselmann, A., Elberich, N., Mathes, J., \& Pietrowsky, R. (2020). Nightmare distress revisited: Cognitive appraisal of nightmares according to Lazarus' transactional model of stress. $J$ Behav Ther Exp Psychiatry, 68, 101517. doi:10.1016/j.jbtep.2019.101517

Gorgoni, M., Scarpelli, S., Alfonsi, V., Annarumma, L., Cordone, S., Stravolo, S., \& De Gennaro, L. (2021). Pandemic dreams: quantitative and qualitative features of the oneiric activity during the lockdown due to COVID-19 in Italy. Sleep Med, 81, 20-32. doi:10.1016/j.sleep.2021.02.006

Groarke, J. M., Berry, E., Graham-Wisener, L., McKenna-Plumley, P. E., McGlinchey, E., \& Armour, C. (2020). Loneliness in the UK during the COVID-19 pandemic: Crosssectional results from the COVID-19 Psychological Wellbeing Study. PLoS One, 15(9), e0239698. doi:10.1371/journal.pone.0239698

Gualano, M. R., Lo Moro, G., Voglino, G., Bert, F., \& Siliquini, R. (2020). Effects of Covid-19 Lockdown on Mental Health and Sleep Disturbances in Italy. Int J Environ Res Public Health, 17(13). doi:10.3390/ijerph17134779

Gupta, M. A. (2020). Spontaneous reporting of onset of disturbing dreams and nightmares related to early life traumatic experiences during the COVID-19 pandemic by patients with posttraumatic stress disorder in remission. J Clin Sleep Med. doi:10.5664/jcsm.8562

Hartmann, E. (1996). Outline for a theory on the nature and functions of dreaming Dreaming, $6(2)$.

Hartmann, E. (2010). The dream always makes new connections: the dream is a creation, not a replay. Sleep Medicine Clinics, 5(2), 241-248.

Hartmann, E., \& Brezler, T. (2008). A systematic change in dreams after 9/11/01. Sleep, 31(2), 213-218. doi:10.1093/sleep/31.2.213

Hedstrom, A. K., Bellocco, R., Hossjer, O., Ye, W., Trolle Lagerros, Y., \& Akerstedt, T. (2021). The relationship between nightmares, depression and suicide. Sleep Med, 77, 1-6. doi:10.1016/j.sleep.2020.11.018

Horton, C. L., \& Malinowski, J. E. (2015). Autobiographical memory and hyperassociativity in the dreaming brain: implications for memory consolidation in sleep. Front Psychol, 6, 874. doi:10.3389/fpsyg.2015.00874

Hovenkamp-Hermelink, J. H. M., Jeronimus, B. F., van der Veen, D. C., Spinhoven, P., Penninx, B., Schoevers, R. A., \& Riese, H. (2019). Differential associations of locus of control with anxiety, depression and life-events: A five-wave, nine-year study to test stability and change. J Affect Disord, 253, 26-34. doi:10.1016/j.jad.2019.04.005

Huang, Y., \& Zhao, N. (2020). Generalized anxiety disorder, depressive symptoms and sleep quality during COVID-19 outbreak in China: a web-based cross-sectional survey. Psychiatry Res, 288, 112954. doi:10.1016/j.psychres.2020.112954

Iorio, I., Sommantico, M., \& Parrello, S. (2020). Dreaming in the Time of COVID-19: A QualiQuantitative Italian Study. Dreaming, 30(3), 199-215. doi:https://doi.apa.org/doiLanding?doi=10.1037\%2Fdrm0000142

Iverach, L., Menzies, R. G., \& Menzies, R. E. (2014). Death anxiety and its role in psychopathology: reviewing the status of a transdiagnostic construct. Clin Psychol Rev, 34(7), 580-593. doi:10.1016/j.cpr.2014.09.002

Kantermann, T. (2020). Behavior: How a Global Social Lockdown Unlocks Time for Sleep. Current Biology, 30(14), R822-R823. doi:10.1016/j.cub.2020.06.037 
Khan, K. S., Mamun, M. A., Griffiths, M. D., \& Ullah, I. (2020). The Mental Health Impact of the COVID-19 Pandemic Across Different Cohorts. Int J Ment Health Addict, 1-7. doi:10.1007/s11469-020-00367-0

Kilius, E., Abbas, N. H., McKinnon, L., \& Samson, D. R. (2021). Pandemic Nightmares: COVID-19 Lockdown Associated With Increased Aggression in Female University Students' Dreams. Front Psychol, 12, 644636. doi:10.3389/fpsyg.2021.644636

Kornilaki, E. N. (2021). The psychological effect of COVID-19 quarantine on Greek young adults: Risk factors and the protective role of daily routine and altruism. Int J Psychol. doi:10.1002/ijop.12767

Lee, S. A., Jobe, M. C., Mathis, A. A., \& Gibbons, J. A. (2020). Incremental validity of coronaphobia: Coronavirus anxiety explains depression, generalized anxiety, and death anxiety. J Anxiety Disord, 74, 102268. doi:10.1016/j.janxdis.2020.102268

Lehmann, S., Skogen, J. C., Haug, E., Mæland, S., Fadnes, L. T., Sandal, G. M., . . Bjørkness, R. (2021). Perceived consequences and worries among youth in Norway during the COVID-19 pandemic lockdown. Scandinavian Journal of Public Health, 1-11. doi:https://doi.org/10.1177/1403494821993714

Leone, M. J., Sigman, M., \& Golombek, D. A. (2020). Effects of lockdown on human sleep and chronotype during the COVID-19 pandemic. Current Biology, 30(16), R930-R931. doi:10.1016/j.cub.2020.07.015

Levin, R., \& Fireman, G. (2002). Nightmare prevalence, nightmare distress, and self-reported psychological disturbance. Sleep, 25(2), 205-212. Retrieved from http://www.ncbi.nlm.nih.gov/pubmed/11902430

Levin, R., \& Nielsen, T. (2007). Disturbed dreaming, posttraumatic stress disorder, and affect distress: a review and neurocognitive model. Psychol Bull, 133(3), 482-528. doi:10.1037/0033-2909.133.3.482

Lin, Y. Q., Zhuang, W. J., Zheng, F. H., Zeng, Z. N., Wu, Y. X., Wu, S. Y., \& Wei, S. C. (2020). Weekday and weekend sleep deprivation are associated with recurrent nightmare in adolescents: a cross-sectional study. Sleep Med, 76, 36-42. doi:10.1016/j.sleep.2020.09.016

MacKay, C., \& DeCicco, T. L. (2020). Pandemic Dreaming: The Effect of COVID-19 on Dream Imagery, a Pilot Study. Dreaming, 30(3), 222-234. doi:https://doi.apa.org/doiLanding?doi=10.1037\%2Fdrm0000148

Malinowski, J. E., \& Horton, C. L. (2014). Memory sources of dreams: the incorporation of autobiographical rather than episodic experiences. Journal of Sleep Research. doi: $10.1111 /$ jsr.12134

Malinowski, J. E., \& Horton, C. L. (2015). Metaphor and hyperassociativity: the imagination mechanisms behind emotion assimilation in sleep and dreaming. Front Psychol, 6, 1132. doi:10.3389/fpsyg.2015.01132

Marelli, S., Castelnuovo, A., Somma, A., Castronovo, V., Mombelli, S., Bottoni, D., ... FeriniStrambi, L. (2020). Impact of COVID-19 lockdown on sleep quality in university students and administration staff. $J$ Neurol. doi:10.1007/s00415-020-10056-6

Marinova, P., Koychev, I., Laleva, L., Kancheva, L., Tsvetkov, M., Bilyukov, R., .. Koychev, G. (2014). Nightmares and suicide: predicting risk in depression. Psychiatr Danub, 26(2), 159-164. Retrieved from http://www.ncbi.nlm.nih.gov/pubmed/24909253

Mautong, H., Gallardo-Rumbea, J. A., Alvarado-Villa, G. E., Fernandez-Cadena, J. C., AndradeMolina, D., Orellana-Roman, C. E., \& Cherrez-Ojeda, I. (2021). Assessment of 
depression, anxiety and stress levels in the Ecuadorian general population during social isolation due to the COVID-19 outbreak: a cross-sectional study. BMC Psychiatry, 21(1), 212. doi:10.1186/s12888-021-03214-1

Monroe, S. M., \& Simons, A. D. (1991). Diathesis-stress theories in the context of life stress research: implications for the depressive disorders. Psychol Bull, 110(3), 406-425. Retrieved from https://www.ncbi.nlm.nih.gov/pubmed/1758917

Monterrosa-Castro, A., Redondo-Mendoza, V., \& Mercado-Lara, M. (2020). Psychosocial factors associated with symptoms of generalized anxiety disorder in general practitioners during the COVID-19 pandemic. Journal of Investigative Medicine . doi:10.1136/jim2020-001456

Mota, N. B., Weissheimer, J., Ribeiro, M., de Paiva, M., Avilla-Souza, J., Simabucuru, G., . . Ribeiro, S. (2020). Dreaming during the Covid-19 pandemic: Computational assessment of dream reports reveals mental suffering related to fear of contagion. PLoS One, 15(11), e0242903. doi:10.1371/journal.pone.0242903

Nielsen, T. (2000). Covert REM sleep effects on REM mentation: Further methodological considerations and supporting evidence. Behav Brain Sci, 23(6), 1040-1057.

Nielsen, T. (2010). Nightmares associated with the eveningness chronotype. J Biol Rhythms, 25(1), 53-62. doi:10.1177/0748730409351677

Nielsen, T. (2017). The Stress Acceleration Hypothesis of Nightmares. Front Neurol, 8, 201. doi:10.3389/fneur.2017.00201

Nielsen, T. (2020). Infectious dreams: how the COVID-19 pandemic is changing our sleeping lives. Scientific American(October 2020), 31-34.

Nielsen, T., \& Lara-Carrasco, J. (2007). Nightmares, Dreaming and Emotion Regulation: A Review. In D. Barrett \& M. McNamara (Eds.), The New Science of Dreaming (Vol. 2, pp. 253-284): Praeger.

Nielsen, T., \& Levin, R. (2007). Nightmares: a new neurocognitive model. Sleep Med Rev, 11(4), 295-310. doi:10.1016/j.smrv.2007.03.004

Nielsen, T., \& Stenstrom, P. (2005). What are the memory sources of dreaming? Nature, 437(7063), 1286-1289. doi:10.1038/nature04288

Nielsen, T., Stenstrom, P., \& Levin, R. (2006). Nightmare frequency as a function of age, gender, and September 11, 2001: Findings from an Internet questionnaire. Dreaming, $16(3)$.

Nielsen, T., Zadra, A., Simard, V., Saucier, S., Stenstrom, P., Smith, C., \& Kuiken, D. (2003). The typical dreams of Canadian university students. Dreaming, 13(4).

Nielsen, T. A., \& Carr, M. (2017). Nightmares and nightmare function. In M. H. Kryger, T. Roth, \& W. Dement (Eds.), Principles and Practices of Sleep Medicine, 6e (pp. 546554): Elsevier.

Pesonen, A. K., Lipsanen, J., Halonen, R., Elovainio, M., Sandman, N., Makela, J. M., ... Kuula, L. (2020). Pandemic Dreams: Network Analysis of Dream Content During the COVID-19 Lockdown. Front Psychol, 11, 573961. doi:10.3389/fpsyg.2020.573961

Pryce, C. R., Azzinnari, D., Spinelli, S., Seifritz, E., Tegethoff, M., \& Meinlschmidt, G. (2011). Helplessness: a systematic translational review of theory and evidence for its relevance to understanding and treating depression. Pharmacology and Therapeutics, 132(3), 242267. doi:10.1016/j.pharmthera.2011.06.006

Rani, V. (2020, October 12 2020). Women are Having More Anxious and Angry Dreams Than Men in the Pandemic, Study Says. Vice. Retrieved from 
https://www.vice.com/en/article/ep4jbw/women-dreams-more-anxious-and-angry-incoronavirus-pandemic

Rek, S., Sheaves, B., \& Freeman, D. (2017). Nightmares in the general population: identifying potential causal factors. Social Psychiatry and Psychiatric Epidemiology, 52(9), $1123-$ 1133. doi:10.1007/s00127-017-1408-7

Roberts, J., Lennings, C. J., \& Heard, R. (2009). Nightmares, life stress, and anxiety: an examination of tension reduction. Dreaming, 19(1), 17-29. doi:https://doi.org/10.1037/a0014787

Robillard, R., Daros, A. R., Phillips, J. L., Porteous, M., Saad, M., Pennestri, M. H., ... Quilty, L. C. (2021). Emerging New Psychiatric Symptoms and the Worsening of Pre-existing Mental Disorders during the COVID-19 Pandemic: A Canadian Multisite Study: Nouveaux symptomes psychiatriques emergents et deterioration des troubles mentaux preexistants pendanturant la pandemie de la COVID-19: une etude canadienne multisite. Can J Psychiatry, 706743720986786. doi:10.1177/0706743720986786

Robillard, R., Dion, K., Pennestri, M. H., Solomonova, E., Lee, E., Saad, M., . . Kendzerska, T. (2020). Profiles of sleep changes during the COVID-19 pandemic: Demographic, behavioural and psychological factors. Journal of Sleep Research, e13231. doi: 10.1111 jsr.13231

Robillard, R., Dion, K., Pennestri, M. H., Solomonova, E., Lee, E., Saad, M., . . Kendzerska, T. (2021). Profiles of sleep changes during the COVID-19 pandemic: Demographic, behavioural and psychological factors. Journal of Sleep Research, 30(1), e13231. doi:10.1111/jsr.13231

Robillard, R., Saad, M., Edwards, J., Solomonova, E., Pennestri, M. H., Daros, A., . . Kendzerska, T. (2020). Social, financial and psychological stress during an emerging pandemic: observations from a population survey in the acute phase of COVID-19. BMJ Open, 10(12), e043805. doi:10.1136/bmjopen-2020-043805

Rosas, M. A., Cordeiro, T. D. C., Silva, T., Caldas, A. S. C., Ribeiro, C., Marinho, M., ... Vasconcelos, S. C. (2019). Self-efficacy assessment tools for individuals with depression: protocol for a systematic review of the literature. BMJ Open, 9(6), e021895. doi:10.1136/bmjopen-2018-021895

Rush, A. J., Trivedi, M. H., Ibrahim, H. M., Carmody, T. J., Arnow, B., Klein, D. N., . . Keller, M. B. (2003). The 16-Item Quick Inventory of Depressive Symptomatology (QIDS), clinician rating (QIDS-C), and self-report (QIDS-SR): a psychometric evaluation in patients with chronic major depression. Biol Psychiatry, 54(5), 573-583. doi:10.1016/s0006-3223(02)01866-8

Salari, N., Hosseinian-Far, A., Jalali, R., Vaisi-Raygani, A., Rasoulpoor, S., Mohammadi, M., . . . Khaledi-Paveh, B. (2020). Prevalence of stress, anxiety, depression among the general population during the COVID-19 pandemic: a systematic review and meta-analysis. Globalization and Health, 16(1), 1-11.

Sandman, N., Valli, K., Kronholm, E., Ollila, H. M., Revonsuo, A., Laatikainen, T., \& Paunio, T. (2013). Nightmares: Prevalence among the Finnish General Adult Population and War Veterans during 1972-2007. Sleep, 36(7), 1041-1050. doi:10.5665/sleep.2806

Scarpelli, S., Alfonsi, V., Mangiaruga, A., Musetti, A., Quattropani, M. C., Lenzo, V., .. . Franceschini, C. (2021). Pandemic nightmares: Effects on dream activity of the COVID19 lockdown in Italy. Journal of Sleep Research, e13300. doi:10.1111/jsr.13300 
Schredl, M., \& Bulkeley, K. (2020). Dreaming and the COVID-19 Pandemic: A Survey in a U.S. Sample. Dreaming, 30(3), 189-198.

Schredl, M., Ciric, P., Gotz, S., \& Wittmann, L. (2004). Typical dreams: stability and gender differences. J Psychol, 138(6), 485-494. doi:10.3200/JRLP.138.6.485-494

Schredl, M., Gilles, M., Wolf, I., Peus, V., Scharnholz, B., Sutterlin, M., .. . Deuschle, M. (2019). Nightmares and Stress: A Longitudinal Study. J Clin Sleep Med, 15(9), 12091215. doi:10.5664/jcsm.7904

Simor, P., Polner, B., Bathori, N., Ortega, R., Van Roy, N., Saenz, A., . . Peigneux, P. (2020). Home confinement during the COVID-19: day-to-day associations of sleep quality with rumination, psychotic-like experiences and somatic symptoms. PsyArxiv Preprint. doi:10.31234/osf.io/wceqt

Spitzer, R. L., Kroenke, K., Williams, J. B., \& Lowe, B. (2006). A brief measure for assessing generalized anxiety disorder: the GAD-7. Arch Intern Med, 166(10), 1092-1097. doi:10.1001/archinte.166.10.1092

Spoormaker, V. I., Schredl, M., \& van den Bout, J. (2006). Nightmares: from anxiety symptom to sleep disorder. Sleep Med Rev, 10(1), 19-31. doi:10.1016/j.smrv.2005.06.001

Stenstrom, P., Fox, K., Solomonova, E., \& Nielsen, T. (2012). Mentation during sleep onset theta bursts in a trained participant: A role for NREM Stage 1 sleep in memory processing? International Journal of Dream Research, 5(1), 37-46.

Strößner, C. (2020). Compositionality Meets Belief Revision: a Bayesian Model of Modification. Review of Philosophy and Psychology, 1-22.

Trnka, R., \& Lorencova, R. (2020). Fear, anger, and media-induced trauma during the outbreak of COVID-19 in the Czech Republic. Psychol Trauma, 12(5), 546-549. doi: $10.1037 / \operatorname{tra} 0000675$

Tu, Z. H., He, J. W., \& Zhou, N. (2020). Sleep quality and mood symptoms in conscripted frontline nurse in Wuhan, China during COVID-19 outbreak: A cross-sectional study. Medicine, 99(26), e20769. doi:10.1097/MD.0000000000020769

Valenti, L. (2020, July 21 2020). Still Having Bad Dreams? Here's What You Can Do About It. Vogue. Retrieved from https://www.vogue.com/article/bad-dreams-lockdowncoronavirus-pandemic

van Rijn, E., Eichenlaub, J. B., Lewis, P. A., Walker, M. P., Gaskell, M. G., Malinowski, J. E., \& Blagrove, M. (2015). The dream-lag effect: Selective processing of personally significant events during Rapid Eye Movement sleep, but not during Slow Wave Sleep. Neurobiol Learn Mem, 122, 98-109. doi:10.1016/j.nlm.2015.01.009

Veissiere, S. P. L., Constant, A., Ramstead, M. J. D., Friston, K. J., \& Kirmayer, L. J. (2019). Thinking through other minds: A variational approach to cognition and culture. Behav Brain Sci, 43, e90. doi:10.1017/S0140525X19001213

Voitsidis, P., Gliatas, I., Bairachtari, V., Papadopoulou, K., Papageorgiou, G., Parlapani, E., ... Diakogiannis, I. (2020). Insomnia during the COVID-19 pandemic in a Greek population. Psychiatry Res, 289, 113076. doi:10.1016/j.psychres.2020.113076

Walker, M. P., \& van der Helm, E. (2009). Overnight therapy? The role of sleep in emotional brain processing. Psychol Bull, 135(5), 731-748. doi:10.1037/a0016570

Wamsley, E. J., \& Stickgold, R. (2010). Dreaming and offline memory processing. Current Biology, 20(23), R1010-1013. doi:10.1016/j.cub.2010.10.045 
Wamsley, E. J., \& Stickgold, R. (2011). Memory, Sleep and Dreaming: Experiencing Consolidation. Sleep Medicine Clinics, 6(1), 97-108. Retrieved from http://www.ncbi.nlm.nih.gov/pubmed/21516215

Wang, J., Zemmelman, S. E., Hong, D., Feng, X., \& Shen, H. (2020). Does COVID-19 impact the frequency of threatening events in dreams? An exploration of pandemic dreaming in light of contemporary dream theories. Consciousness and Cognition, 87, 103051. doi:10.1016/j.concog.2020.103051

Weaver, C. (2020, April 13, 2020). Why Am I Having Weird Dreams Lately? The New York Times. Retrieved from https://www.nytimes.com/2020/04/13/style/why-weird-dreamscoronavirus.html

Wright, K. P., Jr., Linton, S. K., Withrow, D., Casiraghi, L., Lanza, S. M., Iglesia, H., .. . Depner, C. M. (2020). Sleep in university students prior to and during COVID-19 Stayat-Home orders. Current Biology, 30(14), R797-R798. doi:10.1016/j.cub.2020.06.022

Yu, C. K. C. (2008). Typical dreams experienced by Chinese people. Dreaming, 18(1), 1-10. 